\title{
Acromial Fracture Associated with Coracoid Fracture Not Belong to Floating Shoulder : A Case Report
}

Hua-Yu Liu

Army Medical University

Xiang Hua

Army Medical University

Yang Li

Army Medical University

Yu-Feng Zhao

Army Medical University

Si-Ru Zhou ( $\square$ zhousiru1025@163.com )

Army Medical University

\section{Research Article}

Keywords: shoulder, orthopedic trauma, scapula fracture, classification

Posted Date: May 27th, 2021

DOI: https://doi.org/10.21203/rs.3.rs-395335/v1

License: (c) (1) This work is licensed under a Creative Commons Attribution 4.0 International License. Read Full License 


\section{Abstract}

Floating shoulder is no osseoligamentous connection between the humeral shoulder joint with the axial skeleton. The osseoligamentous of shoulder joint connection compose a ring, including clavicle,acromion, coracoid, glenoid fossa, surgical or anatomical scapula neck, acromioclavicular ligament and coracolclavicular ligament. This ring is known as superior shoulder suspensory complex (SSSC). The double disruptions of this ring are recognized to create a potentially unstable anatomical situation. Thus, floating shoulder is also characterized as double or more disruptions of SSSC. Surgical reduction and fixation for the displacement of those fractures at one or more sites is recommended in previous reports. We present 2 cases of patients with acromial and coracoid displaced fractures which belong to a type of the double disruptions of SSSC, there were still osseoligamentous connection between the humeral shoulder joint with the axial skeleton. The patient's shoulder function after nonoperative management was satisfactory.

\section{Introduction}

The floating shoulder is the most complex injury of shoulder girdle, which involves all anatomical structures of the shoulder joint(1-3). The definition of floating shoulder is the scapula neck fracture combined with the clavicular or acromioclavicular fracture, or the acromioclavicular dislocation, or the rupture of the coracoclavicular and coracoacraomial ligament. In short, floating shoulder is no osseoligamentous connection between the humeral shoulder joint with the axial skeleton (4). To better understand the characteristics of shoulder girdle, some studies proposed the theory of the superior shoulder suspensory complex (SSSC) which including clavicle, acromion, coracoid, glenoid fossa, scapula neck, acromioclavicular ligament and coraclclavicular ligament(5). Any double or more disruptions of SSSC creates a unstable anatomical situation in injured shoulder(1). However, the definition and classification of floating shoulder and double disruptions of SSSC remains fully unclear, which leads the treatment of that is controversial $(1,6,7)$. Double disruptions of SSSC are only need to fixation one site theoretically $(5,6)$. Moreover, some of doctors recommended surgery to both fractures sites of floating shoulder, and proposed that the prognosis of operative treatment is significantly better than nonoperative management(8-10). Therefore, specific types of clinical cases may help to explain this issue. We present 2 case of acromial with coracoid displaced fractures, which belong to a type of the double disruptions of SSSC but not be categorized as floating shoulder.

\section{Case 1 Presentation}

A 27 years old man was transferred to our hospital, who complain the pain and dysfunction of right shoulder and arm. He had met with a road traffic accident 11 days ago and sustained fracture of right humeral shaft, multiple ribs fractures (right 1-6 ribs), acromial and coracoid fracture of the right side(Fig. 1A). He had stable autonomous respiration, physical examination revealed pain, swelling, and restriction of movements of right shoulder and arm, no neurovascular injury. He was temporary fixed with plaster and sling and accepted CT scan. Radiographs views showed coracoid fracture (Ogawa Type I, 
Eyres type V) (Fig. 1B), acromial fracture (Ogawa Type II) (Fig. 1C) and humerus shaft fracture (AO 12B2). Displaced fractures of shoulder were belonging to double disruptions of SSSC. This patient was a truck driver, who needed an earlier return to normal shoulder function. We did the surgical intervention after 14 days from the date of the trauma. Firstly, this patient was given general anesthesia and positioned in supine position. The shaft fracture of his humerus was reduced and then fixed with a locking plate through the anterolateral approach. After that, this patient was changed to the lateral decubitus position on the healthy side. The open reduction of acromial fracture was achieved through the superior approach. Acromial fracture was easily reduced and fixed by an anatomical plate. Subsequently, an expose of coracoid fracture was achieved by modified deltopectoral approach and fracture was fixed by two cancellous screws (Fig. 1D). The patient was immobilized in broad arm sling for 3 weeks while allowing active hand and wrist movements. 3 weeks after surgery, intermittent pendulum exercises and passive mobilization were started. 8 weeks after surgery, active shoulder mobilization and rotator cuff strengthening exercises were performed. At the 6 months after injury, the patient had painless full shoulder movement, Shoulder and Hand (DASH(11)) questionnaire recorded 6.7 and Constant (Murley)score CS(12) 93. At the end of follow up (29 mouths after injury), the patient only complained uncomfortable when he were sleep on the right side, but not regularly, DASH recorded 0.8 and CS 99(Fig. 1E-G).

\section{Case 2 Presentation}

A 87 years old man was referred to our hospital complaining of right shoulder and hip pain and dysfunction. He had met with a road traffic accident 4 hours ago. Physical examination revealed pain, swelling, and restriction of movements of right leg and shoulder. He was temporary fixed with traction and sling and accepted X-ray and CT scan (Fig. 2A). Radiographs views showed comminuted coracoid fracture (Ogawa Type I, Eyres type V) (Fig. 2B), acromial fracture (Ogawa Type II) (Fig. 2C) and femoral shaft fracture (AO 32-B2), femoral neck fracture (AO 31-B2). All fractures were displaced. He was stabilized hemodynamically in ICU. Then he was given surgery to femoral neck and shaft 7 days after injury. The shoulder was given non-operative management because of the age. The right up limb was immobilized in broad arm sling for 6 weeks while allowing active hand and wrist movements. 6 weeks after injury, intermittent pendulum exercises and passive mobilization were started. 2 mouths after injury, active shoulder mobilization exercises and rotator cuff strengthening exercises were performed. At the 3 months, this patient was obviously limited at elevation, external and internal rotation, those problem affected his life, DASH questionnaire recorded 21.7 and CS 64. After continuous exercise, the shoulder function gradually improved. At the end of follow up (35 mouths after injury), the patient felt painless and was satisfied with his shoulder function, DASH recorded 6.89 and CS 94(Fig. 2D-F).

\section{Discussion}

Herscovici et al defined floating shoulder as mid-shaft clavicle fracture with scapular-neck fracture In 1993, Goss developed the theory of double disruptions of SSSC which appears to interpret all unstable 
shoulder injury perfectly(5). A number of studies used the term floating shoulder to designate any double disruptions of $\operatorname{SSSC}(6,7)$. To understood easily, SSSC can be divided into three parts(4). Acromial part is consisting of clavicular bony part of acromioclavicular joint, acromioclavicular ligament and acromial process. Coracoid part is consisting of coraclclavicular ligament and coracoid. Neck part is consisting of glenoid fossa and scapula neck. Jan Barton'ıcek et al emphasized injury without a fracture of the anatomical or surgical neck does not result in floating shoulder(4). They considered double disruptions of SSSC without neck fracture are relative stable, because the muscles of the rotator cuff tend to compress the fragments, and those injuries had no impact on displacement or stability of glenohumeral joint, In other word, floating shoulder should exclude the combination of acromial part and coracoid part. Our cases show this type of double disruptions of SSSC which is not belong to floating shoulder.

The injury pattern of our 2 cases were similar. We consider fracture of coracoid and acromion as a result of lateral and horizontal energy which acts on shoulder. Although injuries to those parts of the SSSC affect stability and displacement of acromioclavicular joint and distal clavicle, they have no impact on the stability of glenohumeral joint. Therefore, double disruptions of SSSC include floating shoulder and the injury type of our cases. In other word, injuries of Scapula fracture without articular cartilage injury can be divided into three groups. Single disruption or no disruption of SSSC are considered Type A injuries which should be non-operative (Fig. 3A). Acromial part disruption with coracoid part disruption of SSSC (double disruptions without neck fracture)are considered Type B injuries whose surgical indications are still controversial(Fig. 3B). Floating shoulder (double disruptions including neck fracture)are classified as Type $C$ injuries which need surgery(Fig. 3C).

Coracoid fracture usually fixed with screws, and there was no gold standard to fix single acromial fractures or multiple scapular fractures (13). There were a variety of fixation techniques for single acromial fractures including K-wire fixation, tension band wiring, screw fixation and plate fixation. In case1, anatomical plate for acromial fracture was selected to provide stability. First step of our surgery was the management of acromial fracture, because the surgical exposure and reduction are more easier than operation of coracoid fracture. During the operation, we found that when anatomic reduction of acromial fracture was achieved, the reduction of coracoid fracture can be performed easily. On the other hand, nonoperative management of shoulder fracture includes pain control and sling for 6 weeks. 6 weeks after injury, intermittent pendulum exercises and passive mobilization were started. 2 mouths after injury, active shoulder mobilization exercises and rotator cuff strengthening exercises were performed.

System review of floating shoulder shows that prognosis of patients with surgery is better than those with nonoperative management. However shoulder function of our cases were satisfactory no mater surgery or nonoperative management. Shoulder function of case 1 with surgery was better than shoulder function of case 2 with nonoperative management at the early stage. But at the end of follow up, shoulder function of both cases were satisfactory. Surgery is beneficial only for rapid recovery of shoulder function. 
Unfortunately, scapula fractures were accounting for $1 \%$ of all fractures, acromial with coracoid Fracture more rarely. Westphal T reported only 5 cases in 5 years (between 2009 and 2014)(14). In our trauma centre acromial fracture with coracoid fracture without other SSSC disruption were $1.7 \%$ of all scapular fractures (2 of 117 shoulders)in two years(between December 2016 to December 2018). More cases will needed to show more clinical features of this type of injury.

\section{Declarations}

\section{i. Funding}

This research has received no specific grant from any funding agency in the public, commercial, or notfor-profit sectors.

\section{ii. Conflicts of interest/Competing interests (include appropriate disclosures)}

All authors have disclosed no conflict of interest.

\section{iii. Ethics approval (include appropriate approvals or waivers)}

This clinical study is a retrospective study, which only collects the clinical data of patients, does not interfere with the treatment plan of patients, and will not bring physiological risks to patients. All data and figures were obtained in accordance with ethical requirements according to the ethical guidelines of the Helsinki Declaration. Written informed consent was obtained from individual or guardian participants.

\section{iv. Consent to participate (include appropriate statements)}

Written informed consent was obtained for each participant according to federal and institutional guidelines.

\section{v. Consent for publication (include appropriate statements)}

Written informed consent for publication was obtained from all participants.

\section{vi. of data and material (data transparency)}

All data generated or analysed during this study are included in this published article

\section{vii. Code availability (software application or custom code)}

Not applicable.

\section{viii. Authors' contributions}

All persons who meet authorship criteria are listed as authors, and all authors certify that they have participated sufficiently in the work to take public responsibility for the content, including participation in 
the concept, design, analysis, writing, or revision of the manuscript. Each author certifies that this material or part thereof has not been published in another journal, that it is not currently submitted elsewhere, and that it will not be submitted elsewhere until a final decision regarding publication of the manuscript.

Indicate the specific contributions made by each author.

Category 1

Conception and design of study: Hua-Yu Liu, Si-Ru Zhou;

acquisition of data:Hua-Yu Liu, Xiang Hua, Yang Li,;

analysis and/or interpretation of data: Hua-Yu Liu, Xiang Hua, Yang Li, Yu-Feng Zhao, Si-Ru Zhou;

Category 2

Drafting the manuscript: Hua-Yu Liu, Xiang Hua;

revising the manuscript critically for important intellectual content: Yu-Feng Zhao, Si-Ru Zhou .

Category 3

Approval of the version of the manuscript to be published (the names of all authors must be listed):

Hua-Yu Liu, Xiang Hua, Yang Li , Yu-Feng Zhao, Si-Ru Zhou.

\section{References}

1. Owens BD, Goss TP. The floating shoulder. J Bone Joint Surg Br. 2006;88(11):1419-24.

2. Lambert S, Kellam JF, Jaeger M, Madsen JE, Babst R, Andermahr J, et al. Focussed classification of scapula fractures: failure of the lateral scapula suspension system. Injury. 2013;44(11):1507-13.

3. Pailhes RG, Bonnevialle N, Laffosse J, Tricoire J, Cavaignac E, Chiron P. Floating shoulders: Clinical and radiographic analysis at a mean follow-up of 11 years. Int J Shoulder Surg. 2013;7(2):59-64.

4. Bartonicek J, Tucek M, Nanka O. Floating Shoulder: Myths and Reality. JBJS Rev. 2018;6(10):e5.

5. Goss TP. Double disruptions of the superior shoulder suspensory complex. J Orthop Trauma. 1993;7(2):99-106.

6. Oh W, Jeon IH, Kyung S, Park C, Kim T, Ihn C. The treatment of double disruption of the superior shoulder suspensory complex. Int Orthop. 2002;26(3):145-9.

7. Friederichs J, Morgenstern M, Buhren V. Scapula fractures in complex shoulder injuries and floating shoulders: a classification based on displacement and instability. J Trauma Manag Outcomes. 2014;8:16.

8. Bartonicek J, Fric V, Tucek M. Fractures of the anatomical neck of the scapula: two cases and review of the literature. Arch Orthop Trauma Surg. 2013;133(8):1115-9. 
9. Kim KC, Rhee KJ, Shin HD, Yang JY. Can the glenopolar angle be used to predict outcome and treatment of the floating shoulder? J Trauma. 2008;64(1):174-8.

10. Lin TL, Li YF, Hsu CJ, Hung CH, Lin CC, Fong YC, et al. Clinical outcome and radiographic change of ipsilateral scapular neck and clavicular shaft fracture: comparison of operation and conservative treatment. J Orthop Surg Res. 2015;10:9.

11. Chen H, Ji XR, Zhang W, Zhang YL, Zhang LH, Tang PF. Validation of the simplified Chinese (Mainland) version of the Disability of the Arm, Shoulder, and Hand questionnaire (DASHCHNPLAGH). Journal of Orthopaedic Surgery and Research. 2015;10:76.

12. Constant CR, Gerber C, Emery RH, Solbjerg JO, Boileau P. A review of the Constant score: Modifications and guidelines for its use. J Shoulder Elb Surg. 2008;17(2):355-61.

13. Dombrowsky AR, Boudreau S, Quade J, Brabston EW, Ponce BA, Momaya AM. Clinical outcomes following conservative and surgical management of floating shoulder injuries: a systematic review. J Shoulder Elbow Surg. 2020;29(3):634-42.

14. Westphal T, Lippisch R, Jurgens J, Piatek S. [Simultaneous fracture of the acromion and coracoid process : Rare variant of double disruption of the superior shoulder suspensory complex]. Unfallchirurg. 2018;121(12):968-75.

\section{Figures}



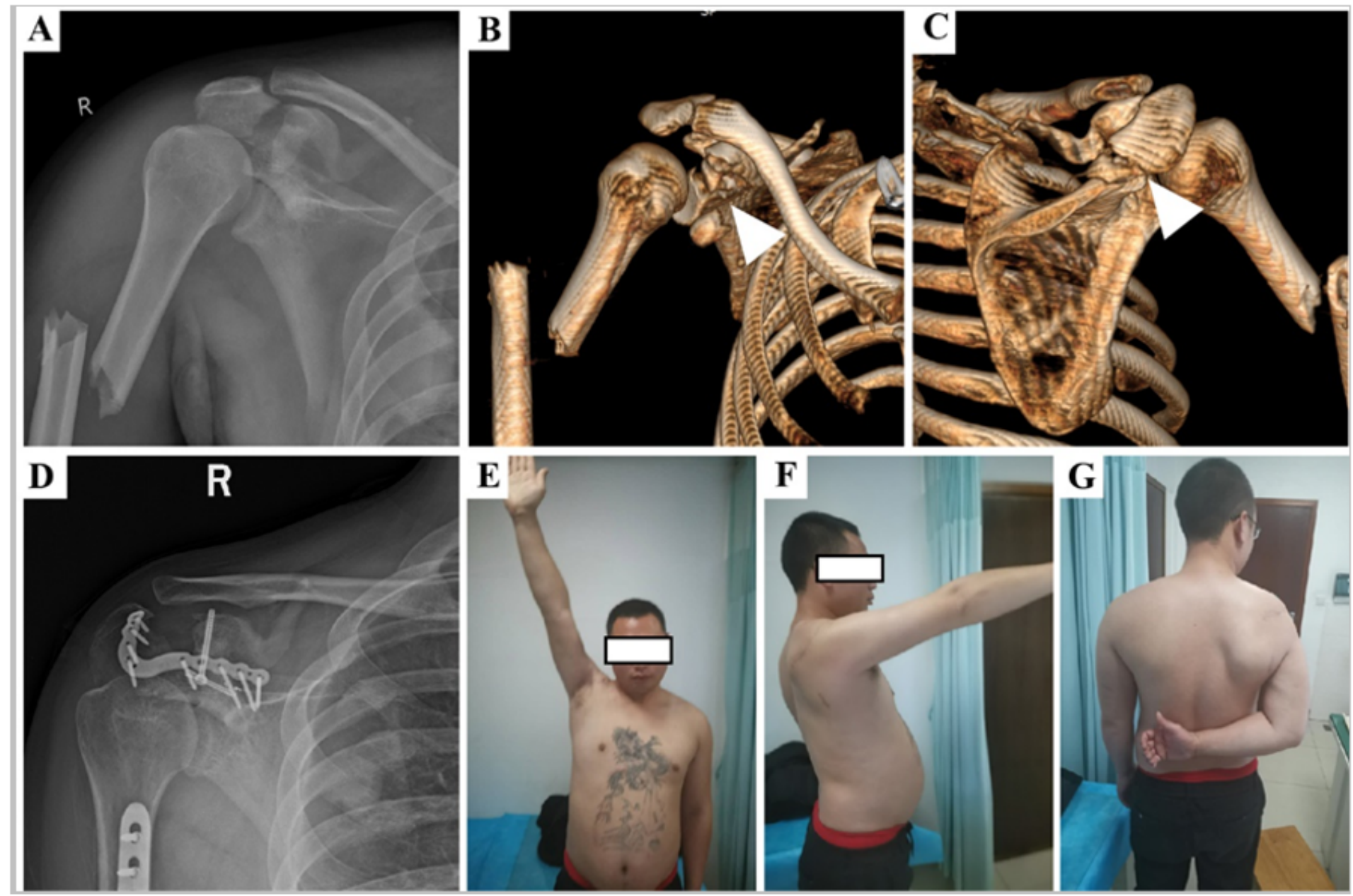

\section{Figure 1}

A patient with acromial and coracoid fractures was given surgery. The 27 male was treated with osteosynthesis of the right scapula after a road traffic accident. At the 6 months after injury, the patient had painless full shoulder movement. At the end of follow up (29 mouths after injury), the patient only complained uncomfortable when he sleep on the right side, but not regularly. A Preoperative X-ray of his right shoulder in AP view showing the displaced acromial and coracoid fracture. B Preoperative CT scan of his right shoulder showing displaced fracture of the coracoid (white arrow).C Preoperative CT scan of his right shoulder showing displaced fracture of the acromion (white arrow).D Postoperative AP view showing union at 6 mouths follow up. E-G Full range of shoulder motion of this patient at 29 months follow up. 

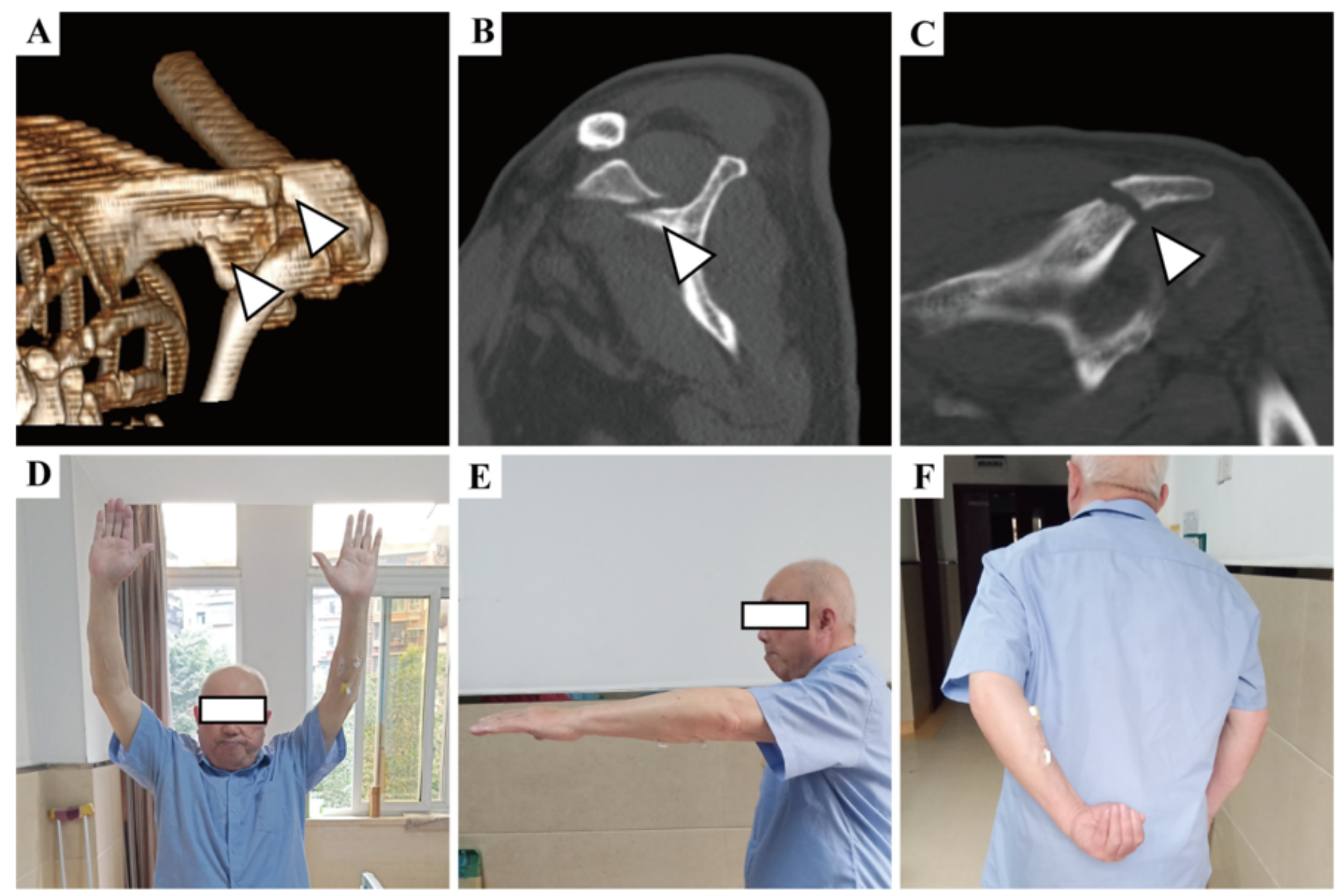

\section{Figure 2}

The patient with acromial and coracoid displaced fractures was given non-operative management. A 87 male was met with a road traffic accident and sustained a acromial and coracoid fractures. He was treated with pain control and sling for 6 weeks. Passive mobilization exercises were started in the 6 weeks. Active and strengthening exercises were performed in 2 mouths after injury. At 3 months after injury, the patient was obviously limited at elevation, external and internal rotation. After continuous exercise, the shoulder function gradually improved. At the end of follow up (29 mouths after injury),the patient felt painless and was satisfied with his shoulder function. A Right shoulder CT scan reconstruction showing displaced acromial fracture and coracoid fracture. B Right shoulder CT scan showing displaced coracoid fracture. C CT scan showing displaced fracture of the acromial fracture (white arrow). D-F Range of shoulder motion of this patient at 35 months follow up. 


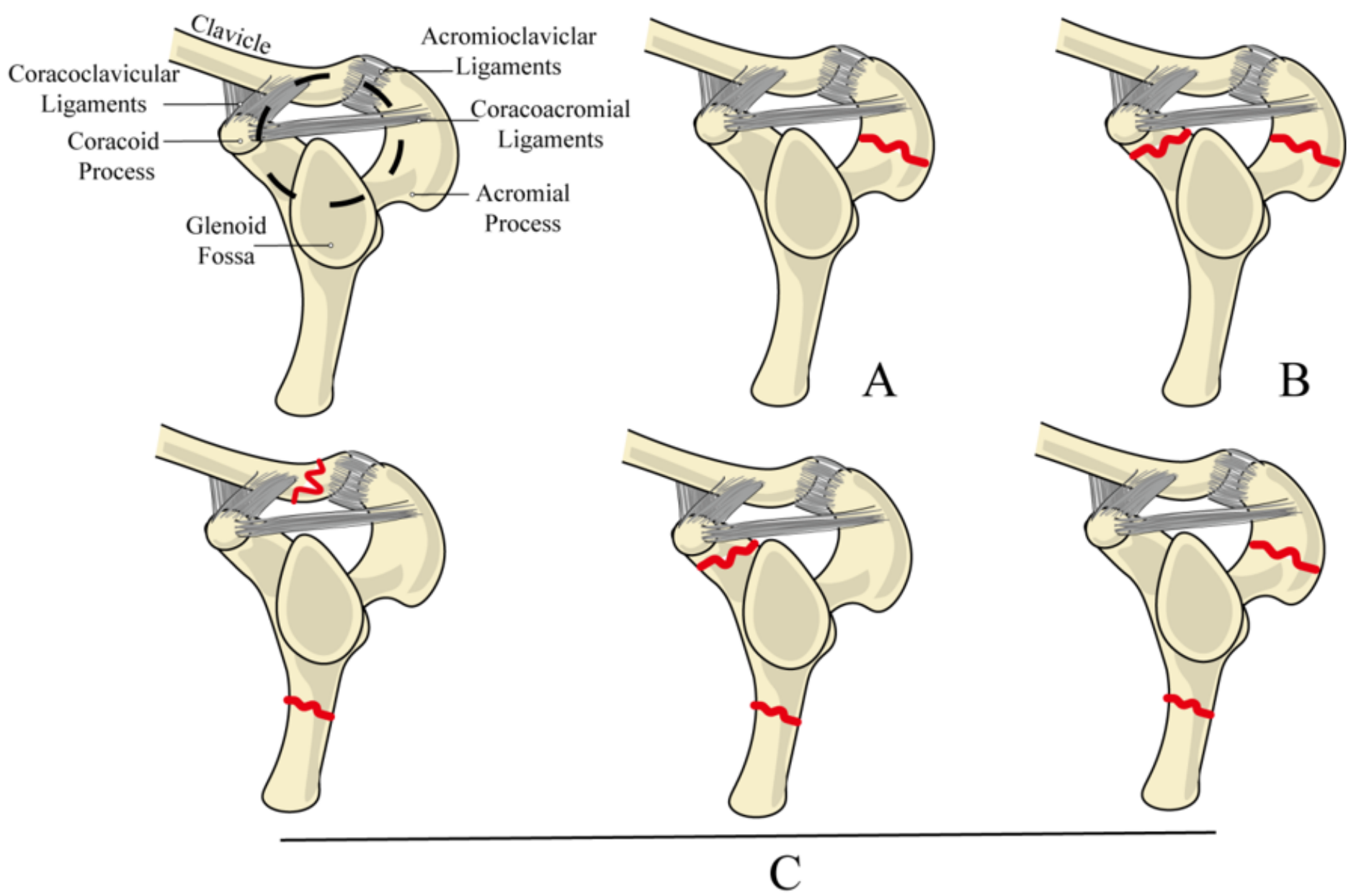

Figure 3

Classification of Scapula fracture without articular cartilage injury. A Single disruption or no disruption of SSSC are classified as type A injuries which should be non-operative. B Double disruptions of SSSC without neck fracture (acromial part disruption with coracoid part disruption) are classified as type $B$ injuries whose surgical indications are still controversial. C Double disruptions including neck fracture (floating shoulder) are classified as type $\mathrm{C}$ injuries which need surgery. 